\title{
SOCIEDAD DE CIRUGÍA DE BOGOTÁ: EL MUSEO Y LA REVISTA REPERTORIO*
}

\section{REINAUGURACIÓN DE LA EXPOSICIÓN PERMANENTE}

\author{
María Antonieta García Restrepo**
}

Con motivo del aniversario ciento siete (107) de la Sociedad de Cirugía de Bogotá fundada el 22 de julio de 1902, el museo de la Sociedad de Cirugía de Bogotá reinaugura su exposición permanente, que si bien estaba exhibida en parte desde hace varios años, ahora cuenta con un guión curatorial y apoyos museográficos que acompañan el recorrido de los visitantes por la historia a través de su colección. Este museo tiene como misión construir un escenario de reconocimiento de la historia de la ciencia médica en Colombia, conservando los testimonios materiales, facilitando la investigación de colecciones y promoviendo el conocimiento relacionado con la historia de la medicina y de las instituciones que han participado en su desarrollo. Entre otras actividades el museo ha realizado hasta el momento las siguientes exposiciones: "La medicina en el arte prehispánico colombo-ecuatoriano", "Enanismo en el arte", "El bocio endémico en Colombia", "Parto y lactancia en el arte colombiano", "Enfermedad mental y arte en Colombia" y el "Primer encuentro de museos médicos". Gracias a estas actividades y como fruto del trabajo de estos años, fue posible la reinauguración que partió de elaborar un guión curatorial, que junto con la transformación del museo hicieron de la colección permanente un lugar apropiado para hacer evidente la relación entre cultura y medicina.

\section{Extracto del guión curatorial}

\section{Hugo Sotomayor Tribín MD**}

En el período que va de 1902 a 1925, Bogotá sufrió una importante transformación arquitectónica en la que participaron distinguidos ingenieros y arquitectos nacionales y extranjeros. Entre los primeros figura el ingeniero Diódoro Sánchez, autor de los planos iniciales del Hospital de San José, que incluían un solo piso, adaptados a un terreno muy pequeño, entregados en la ceremonia de la colocación de la primera piedra el 14 de agosto de 1904. De los segundos es figura central el italiano Pietro Cantini, arquitecto de obras importantes como el Teatro Colón y el Capitolio Nacional, autor de los segundos planos y definitivos, con una segunda planta, y responsable de la construcción hasta su inauguración. El maestro constructor fue el señor José Mendoza, quien coordinó las cuadrillas de obreros y albañiles. El arquitecto Cantini como hijo de la época de transición entre la vieja ideología médica de los miasmas de finales del siglo XIX y la moderna de los gérmenes, tomó como modelo para su plano importantes estilos de hospitales europeos construidos a finales del siglo XIX y principios del XX. Estos modelos explican el sistema de pabellones y la altura con la circu-

* Extractos de las intervenciones con motivo de la reinauguración de la exposición permanente, el 22 de julio de 2009. Cuando se inauguró el Hospital de San José, (1925) la Revista Médica de la Academia Nacional de Medicina circulaba desde 1873 y la revista Repertorio de Medicina y Cirugía de la Sociedad de Cirugía de Bogotá desde 1909.

** Coordinadora de colecciones. Museo de la Sociedad de Cirugía de Bogotá DC.

*** Curador del museo, Sociedad de Cirugía de Bogotá, Hospital de San José. Bogotá DC. 
lación del aire al interior de éstos, ya que seguían el sistema de construcción Tollet, que contaba con innovaciones como darle a los techos forma ojival mediante armaduras de hierro sencillas conectándolas con los muros y evitando el uso de tirantes, lo cual favorecía la ventilación además de ser un sistema más económico.

El Hospital de San José inició sus actividades cuando ya se utilizaban en Bogotá los rayos X. Las especialidades quirúrgicas y médicas estaban en ciemes, las técnicas de laboratorio clínico y fisiológico se desplegaban cada vez más y la farmacia todavía era muy dependiente de las boticas y los procesos artesanales. Tras la inauguración del Hospital de San José y durante los tres primeros años de su funcionamiento, la atención de enfermería de los pacientes fue asumida por los Hermanos Hospitalarios de San Juan de Dios. Ellos fueron remplazados a finales de 1928 por las Hermanas de la Presentación. Desde su inauguración hasta el presente, el Hospital de San José ha sido escenario de primera plana del desarrollo de la cirugía, la medicina, la enfermería y la instrumentación médico- quirúrgica en Bogotá y Colombia.

La exposición actual estará exhibida en forma permanente y se complementará como se ha venido haciendo con las exposiciones temporales y demás actividades que integren y diversifiquen la información ofrecida. Queremos hacer del museo el punto de encuentro histórico e institucional, un lugar cada vez más activo, lleno de experiencias, tesoros e historias. Por esto, invitamos a todos a conocerlo. Está ubicado en la primera planta del auditorio Guillermo Fergusson dentro del Hospital de San José, abierto de lunes a viernes de 7 a.m. a 5 p.m.

\section{Lecturas recomendadas}

A.A.V.V. Atlas Histórico de Bogotá. 1539 - 1910. Corporación la Candelaria. Biblioteca Luis Angel Arango. Biblioteca Virtual 2000.

Bermúdez Andrés MD. Informe Presentado por el Presidente de la Sociedad de Cirugía de Bogotá 1930-1931. Revista Repertorio de Medicina y Cirugúa, Edición Especial 2002.

Cadena Rey D. Reseña histórica. Repert medcir. 2002; edición especial (Centenario Sociedad de Cirugía de Bogotá Hospital de San José, 1902-2002).

El debate sobre el Hospital de San José. Bogotá: Editorial Manrique; 200?

Forero Gutiérrez A. Memorias de mi vida. Bogotá: Editorial Kelly; 1958.

Historia de Bogotá. $2^{\mathrm{a}}$ ed. Bogotá: Villegas Editores, 1988 (impresion de 2007). 3 v.

Muñoz L. Historia del Hospital de San José 1902 - 1956. Bogotá: Academia Nacional de Medicina; 1958.

Pérez ME. Remodelación del Hospital de San José de Bogotá [tesis]. Bogotá: Universidad de los Andes. Facultad de Arquitectura; 1938.

Sánchez Diódoro. Informe "Relación Sucinta pero documentada sobre el origen de la obra”. Bogotá 1908. Imprenta Eléctrica Hospital de San José.

Sociedad de Cirugía de Bogotá. Itinerario histórico desde el 22 de julio de 1902. Santafé de Bogotá : Sociedad de Cirugia de Bogota, 1997. 239 p.

Villalobos MC. Informe final: investigación histórica Museo de la Sociedad de Cirugía de Bogotá - Hospital de San José - Fundación Universitaria de Ciencias de la Salud. Bogotá, 2008.

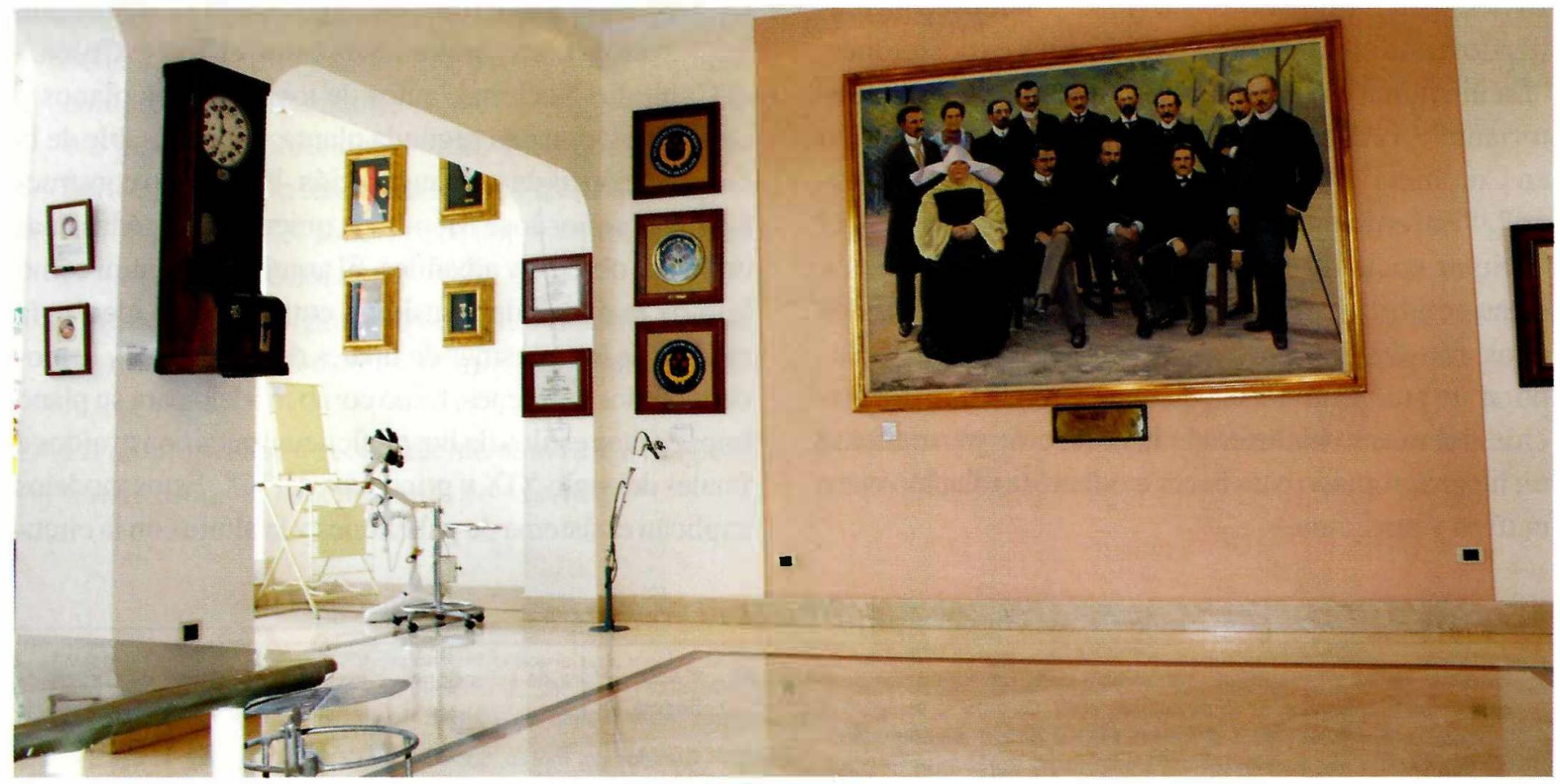

Óleo de los Fundadores en el Museo. 


\section{Aspectos del Museo}
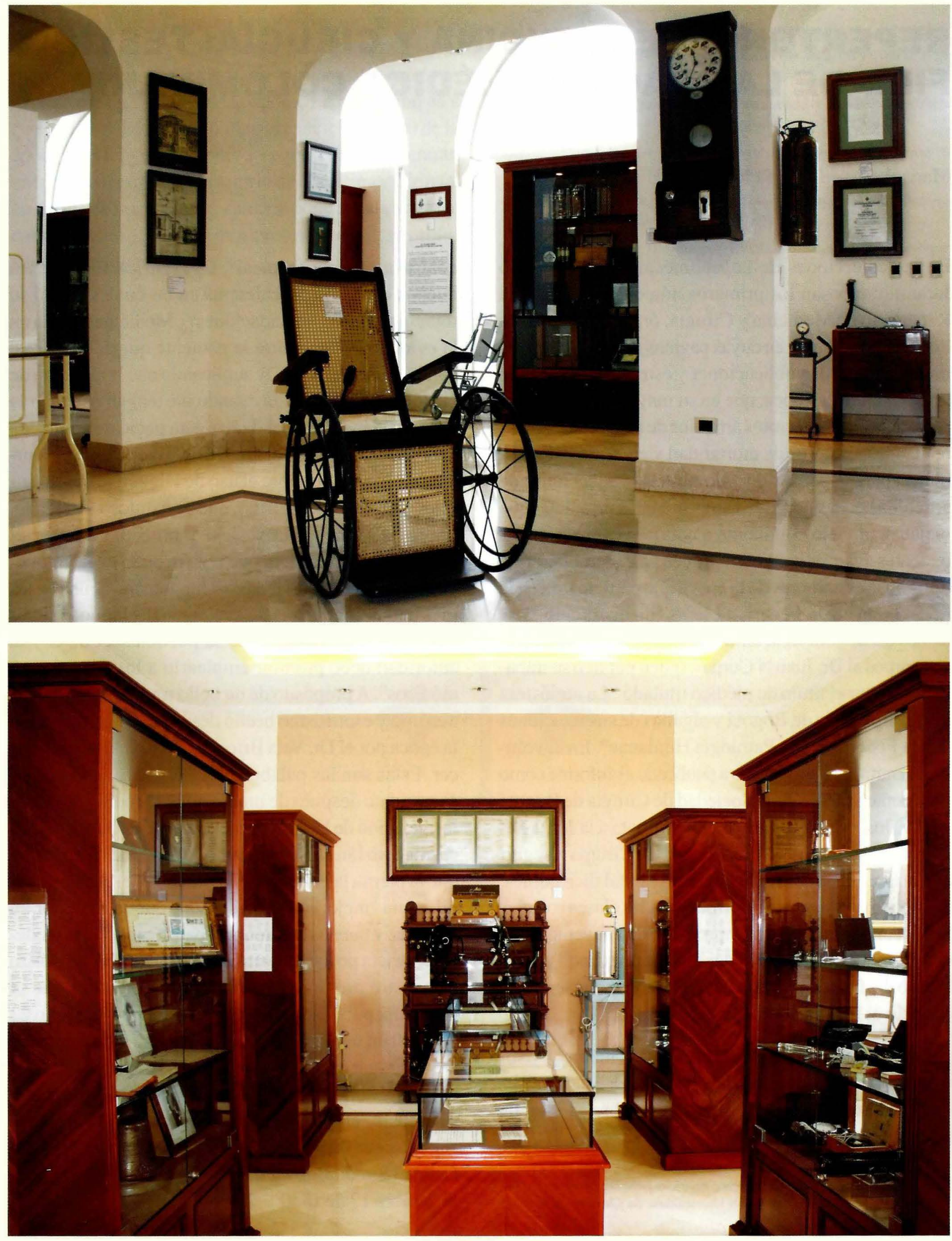